Repository of the Max Delbrück Center for Molecular Medicine (MDC) Berlin (Germany)

http://edoc.mdc-berlin.de/

Sleeping Beauty hits them all: transposon-mediated saturation mutagenesis in the mouse germline

Zsuzsanna Izsvák and Zoltán Ivics 


\title{
Sleeping Beauty hits them all: transposon-mediated saturation mutagenesis in the mouse germline
}

\author{
Zsuzsanna Izsvák ${ }^{1}$ and Zoltán Ivics ${ }^{1}$ \\ ${ }^{1}$ Max Delbrück Center for Molecular Medicine, Berlin, Germany
}

ABSTRACT | The Sleeping Beauty (SB) transposon emerged as a useful tool for applications such as germline and somatic cell insertional mutagenesis and now shows its usefulness again by facilitating saturating germline mutagenesis in mice.

One way of identifying gene function is to bring physical alterations into the gene sequence. A classical way of such an approach is mutagenesis using chemicals or radiations. These agents can be extremely powerful in mutagenizing the DNA, but the 'slow' part is finding the mutated gene itself. If, however, the mutagenizing agent can tag the site of the mutation, the gene identification becomes extremely simple. Transposons can cause mutations by inserting into genes and can be used as tags to fish out the mutated gene. Insertional mutagenesis using transposons have revolutionized genetic screening in flies, worms and plants. What made the adaptation of this approach to vertebrates possible was the creation of the SB transposon system [1]. In this issue, Keng et al. [2] describe major developments in mouse germline mutagenesis using $S B$.

Transposable elements have been isolated from several vertebrate species, but nearly all of the native elements were found to be inactive, indistinguishable from 'junk' DNA. A molecular archaeological approach was used to reconstitute an ancient transposon that was presumably inactivated several million years ago in fish genomes [1]. The revived transposon was named Sleeping Beauty, after the Grimm brothers' famous fairy tale. SB contains a single gene encoding a transposase protein that mediates the jumping, which is flanked by a pair of terminal inverted repeats containing transposase binding sites. In the laboratory, these cis- and trans-acting factors are separated into two vectors that provide the researcher with the ability to control transposition.

Since its debut in 1997, SB has been shown to transpose efficiently in both somatic tissues [3] and the germline[4,5] of the mouse in vivo, and was successfully introduced as a tool for vertebrate genomics [6]. For germline mutagenesis in the mouse, a strain containing a pool of chromosomally integrated $S B$ transposons equipped with mutagenic gene-trap cassettes and poly(A)-trap reporter cassettes (Fig.1a,b) is crossed with a second strain stably expressing the $S B$ transposase. Transposition events that occurred in the male germline of the double transgenic animals can be recovered by crossing with wild-type females. Then one can screen the offspring for activation of the gene-trap reporters, indicative of transposition into genes. The integration loci can then be easily identified, and phenotypic changes associated with the mutations can be examined in homozygous animals. The lessons learned so far from such experiments are that most of the offspring will contain, on average, 1-2 new transposition events, that suitably engineered $S B$ transposons can indeed be used as insertional mutagens and that new transposon insertions tend to cluster around the original transposon donor locus, a phenomenon termed 'local hopping'.
Keng et al. [2] took advantage of SB's local hopping behavior to provide proof-of-concept that all genes within a certain window around a transposon donor locus can be mutagenized by transposon insertions. The authors concentrated their efforts on two transposon donor sites present on different chromosomes in the mouse genome, and then determined whether predicted genes within a 4$\mathrm{Mb}$ window around these sites were hit by transposons. Using a combination of screening based on activation of a poly(A)-trap GFP reporter (Fig.1b) and PCR on DNA samples prepared from germ cells, they established that transposon technology can be used to mutagenize mouse genes at a saturation level within a certain chromosomal interval. Notably, the transposon insertions can clearly be mutagenic, as phenotypes were readily obtained from some of the mice bred to homozygosity.

The major advantage of transposon-mediated insertional mutagenesis in the mouse is the ability to easily generate and maintain whole libraries of insertional mutants in vivo in the testes of founder animals. One can then easily analyze these mutations by simple breeding of the founders. Therefore, the classical embryonic stem cell-germline chimera-mutant animal route is simply skipped, considerably reducing the time and cost associated with production of mutants. To take full advantage of local hopping for saturation mutagenesis, libraries of transposon donors in chromosomal regions of interest (for example, quantitative trait loci or syntenic regions of certain disease loci in which genes of interest are located in clusters) will have to be generated in the future. The Keng et al. loss-of-function mutagenesis in the germline complements strategies that generate gain-offunction dominant phenotypes in the soma. Two recent papers in Nature demonstrate how $S B$ can be used for gain-of-function mutagenesis in somatic cells in mice for tumor gene discovery $[7,8]$.

Both Collier et al. [7] and Dupuy et al. [8] engineered an $S B$ transposon to have the ability to both enhance the expression of genes around the insertion sites and/or to mutate them by transposon insertion. Insertion near or within a gene causes an increase in gene product owing to a viral enhancer and promoter inside the transposon (Fig.1c). This approach is designed to identify oncogenes that promote cancer development. Destruction of genes by physically inserting into them aims to identify tumorsuppressor genes that normally protect against cancer. To overcome the relative inefficiency of the $S B$ system in somatic cells, Collier et al. performed their screen in the p19Arf genetic background that is highly susceptible to cancer, whereas Dupuy et al. used improved components of the $S B$ system that significantly increased transposition frequencies. Notably, in Dupuy's screen, the tumors were induced in wild-type animals, manifesting the feasibility and power of using SB transposon technology in genetic 
backgrounds that do not have a cancer-predisposing mutation. A major breakthrough of the $S B$ somatic mutagenesis screens is that, compared to the earlier retrovirus-mediated mutagenesis screens, new types of cancer genes and gene networks now seem to be identifiable, in essentially any tissue type.

The usefulness of $S B$ in both loss-of-function and gain-offunction screens can be further developed, as the transposon can be designed to be mobilized selectively in a specific tissue type or developmental stage. Bringing conditionality into screens will allow to uncover otherwise lethal mutations, and will permit reversion of phenotypes. For example, inclusion of a Cre-loxP component in the transposon will allow for the removal of the gene-trapping portion of the $S B$ vectors from specific gene insertions (D.A. Largaespada, personal communication). Combination of transposon insertions with Cre-loxP technology might also be exploited for the deletion or inversion of chromosomal regions between chromosomally integrated transposon copies. Saturation mutagenesis can also be approached in the future by using different transposable elements in combination. A battery of DNA transposons similar to $S B$ in terms of structure and mechanism of transposition, as well as retrotransposons such as the L1 element [9] will undoubtedly increase our abilities for insertional mutagenesis and functional annotation of large sets of genes, in part owing to their different preferences for DNA sequences for integration. Finally, screens similar in design to those described here will likely prove realistic for other species, including fish and frogs. With the genome sequences of these organisms nearly completed, transposon technology is bound to revolutionize genomic research in vertebrates.

\section{Corresponding Author}

Zoltán Ivics, zivics@mdc-berlin.de

\section{References}

1. Ivics, Z., Hackett, P.B., Plasterk, R.H. \& Izsvak, Z. Cell 91, 501-510 (1997)

2. Keng, V.W. et al. Nat. Methods 2, 763-769 (2005).

3. Yant, S.R. et al. Nat. Genet. 25, 35-41 (2000).

4. Horie, K. et al. Mol. Cell. Biol. 23, 9189-9207 (2003).

5. Carlson, C.M. et al. Genetics 165, 243-256 (2003).

6. Miskey, C., Izsvak, Z., Kawakami, K. \& Ivics, Z. Cell. Mol. Life Sci. 62, 629-641 (2005).

7. Collier, L.S., Carlson, C.M., Ravimohan, S., Dupuy, A.J. \& Largaespada, D.A. Nature 436, 272-276 (2005).

8. Dupuy, A.J., Akagi, K., Largaespada, D.A., Copeland, N.G. \& Jenkins, N.A. Nature 436, 221-226 (2005).

9. Han, J.S. \& Boeke, J.D. Nature 429, 314-318 (2004). 


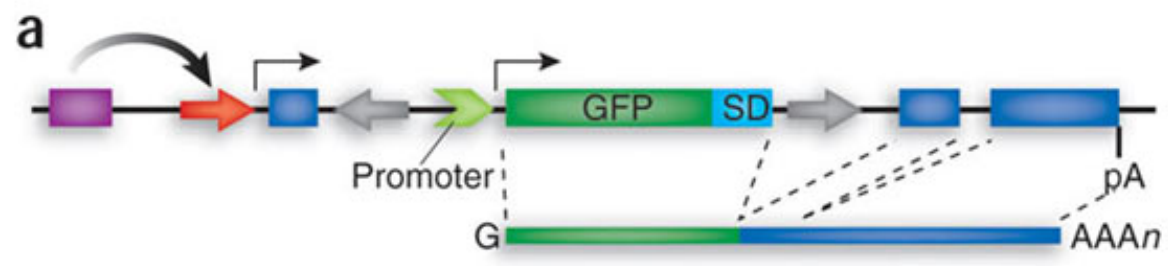

Poly(A) trap: reports insertion into a gene

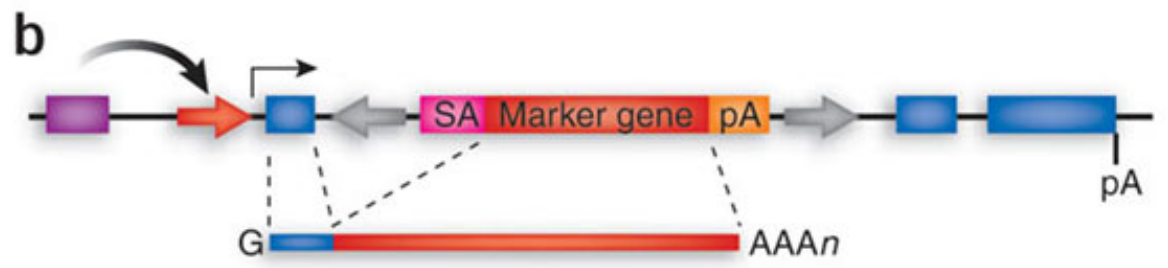

Gene trap: reports insertion into an expressed gene, and disrupts its transcription

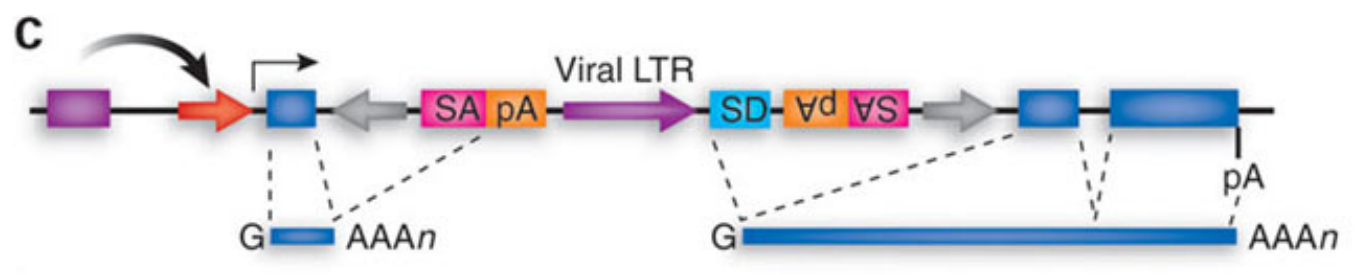

Oncogene trap: disrupts gene transcription and overexpresses a fragment of the normal gene product

Fig.1: Transposon-based vectors.

The type of spliced transcript resulting from a transposition event depends on the transposon-based trapping construct. The three major classes of trapping vectors (delimited by the transposon inverted terminal repeats indicated by gray arrows) and the transcripts resulting from their insertion are depicted in the context of a hypothetical transcriptional unit composed of an upstream regulatory element (purple box), a promoter (red arrow), three exons (dark blue boxes) and a polyadenylation signal (pA). SD and SA represent splice donor and splice acceptor sites, respectively. 